\title{
Determination of Scan-Plane Motion Using Speckle Decorrelation: Theoretical Considerations and Initial Test
}

\author{
Jian-Feng Chen, ${ }^{\star}$ J. Brian Fowlkes, Paul L. Carson, Jonathan M. Rubin \\ Department of Radiology, University of Michigan Medical Center, 200 Zina Pitcher Place, \\ Ann Arbor, Ml 48109-0553
}

Received 20 August 1996; revised 26 August 1996

\begin{abstract}
The correlation function of the echo signal intensities at a fixed region on a series of B-mode images is directly related to the change of speckle patterns between these images. An indication is given here of how the rate of the change of that correlation function can be used to estimate the scan-plane motion in any direction relative to the imaged tissue or other material. In this first implementation it is assumed that the statistical properties of the echo signals follow those of a complex circular Gaussian, and the case is considered of diffusely scattering tissue with many fine particles per resolution cell and with no phase distortion. The method is applied to data from a one-dimensional linear array and initial results are presented for scanning a tissue-mimicking phantom in the elevational direction of the transducer. Experimental results are in good agreement with the predictions. The current method should provide a good indication of the local rate of scan-head motion in those tissues in which the normalized correlation function of the echo signal intensities behaves, or can be made to behave, as it would for a medium with uniform acoustic properties and containing many, randomly distributed, pointlike scatterers, although application might be made to other situations where a deterministic condition exists for the correlation between images taken at differing locations within a volume. (C) 1997 John Wiley \& Sons, Inc. Int J Imaging Syst Technol, 8, 38-44, 1997
\end{abstract}

Key words: ultrasound; 3D; motion estimation; speckle; decorrelation

\section{INTRODUCTION}

The purpose of this article was to study scan-plane motions through tissue and the estimation of their relative positions by analysis of the correlation of speckle patterns in ultrasonic images. Conventionally, second-order statistics have been used to study the correlation of speckle patterns at different locations within ultrasonic images for tracking the relative motion of blood or other moving tissue [1]. Speckle correlation has also been studied for the purpose of speckle reduction for improving lesion

Correspondence to: J. B. Fowlkes

* Present address: Siemens Medical Systems, Inc., P.O. Box 7002, Issaquah, WA $98027-7002$

Contract grant sponsor: USPHS; Contract grant number: RO1 CA55076 $5-4144$ detectability via spatial and frequency compounding [2-5]. These speckle reduction methods involve the averaging of images in which the speckle pattern has been changed. Recently, assessment of blood flow and, potentially, perfusion has been investigated by use of decorrelation of the Doppler signal with time at one location in the image [6]. In this article we use the speckle correlation between corresponding points in a series of scan planes for an alternative purpose: to determine the relative scan-plane positions, orientations, and motions. This may be important for the three-dimensional registration of ultrasonic images, or the detection and correction for tissue motion.

The speckle pattern on conventional B-mode images is caused by the phase-sensitive detection of the scattering from random inhomogeneities in the acoustic properties of biological tissue in the resolution cell of the transducer. When the scan plane is moved slowly, the intensities of time domain echo signals at one distance along one echo line changes, producing a change in the speckle pattern observed at that location on the B-mode images. We analyze the second-order statistical properties of the echo signal intensity from a given depth as a function of time or image frame number, instead of tracking the speckle patterns in subsequent images as a function of position within the images. The second-order statistics are defined as the expectation of the product using pairs of values (intensities) measured at two positions in space, number of frames or times.

In this article, it is assumed that the statistical properties of echo signals follow those of a complex Gaussian. The case considered is of diffusely scattering tissue with many fine particles per resolution cell [2] and with no phase distortion [7]. A method is proposed in which the correlation function of echo signal intensities are used to determine the relative positions of scan planes for motions in the elevational direction. That is the direction, normal to the scan plane, in which the same tissue volume cannot be tracked in the image. The method is applied to data from a modern 1D array imaging system and initial results are presented for 3D scanning of a tissue-mimicking phantom.

\section{THEORY}

A. The Correlation of Speckle Patterns in 3D B-Mode Images. The speckle pattern in B-mode images is directly related 
to spatial variation of the echo signal intensities or their magnitudes. The correlation function of the intensities for a purely random (diffusely scattering) case can be estimated from the correlation function of the complex echo signals. The correlation of the echo signal intensities between two specific positions separated by $\Delta \mathbf{r}$, e.g., $\mathbf{r}$ and $\mathbf{r}+\Delta \mathbf{r}$, is defined as

$$
R(\Delta \mathbf{r})=\langle I(\mathbf{r}) I(\mathbf{r}+\Delta \mathbf{r})\rangle
$$

where $I(\mathbf{r}) \equiv|U(\mathbf{r})|^{2}$ and $I(\mathbf{r}+\Delta \mathbf{r}) \equiv|U(\mathbf{r}+\Delta \mathbf{r})|^{2}$ are the intensities of the echo signals at two specific points, $\mathbf{r}$ and $\mathbf{r}+$ $\Delta \mathbf{r} . U(\mathbf{r})$ and $U(\mathbf{r}+\Delta \mathbf{r})$ are the complex echo signals. $\langle\cdots\rangle$ stands for the expectation of a random variable. The Appendix provides a general derivation for the results which is not dependent on the use of intensity data.

Using the moment theorem for the jointly zero mean, Gaussian random variables, and assuming that the real and imaginary parts of the complex echo signal $U(\mathbf{r}), U_{r}(\mathbf{r})$ and $U_{i}(\mathbf{r})$, are uncorrelated, e.g.,

$$
\left\langle U_{r}(\mathbf{r}) U_{i}(\mathbf{r}+\Delta \mathbf{r})\right\rangle=\left\langle U_{r}(\mathbf{r}+\Delta \mathbf{r}) U_{i}(\mathbf{r})\right\rangle=0,
$$

we have [8]

$$
\begin{aligned}
R(\Delta \mathbf{r})=2\left\langle U_{r}^{2}(\mathbf{r})\right\rangle\left\langle U_{r}^{2}(\mathbf{r}+\Delta \mathbf{r})\right\rangle+4\left\langle U_{r}(\mathbf{r}) U_{r}(\mathbf{r}+\Delta \mathbf{r})\right\rangle^{2} & \\
+ & 2\left\langle U_{i}^{2}(\mathbf{r})\right\rangle\left\langle U_{i}^{2}(\mathbf{r}+\Delta \mathbf{r})\right\rangle .
\end{aligned}
$$

Using the symmetry of the correlation function of the complex echo signals $U(\mathbf{r})$,

$$
\rho(\Delta \mathbf{r})=\frac{\left\langle U(\mathbf{r}) U^{*}(\mathbf{r}+\Delta \mathbf{r})\right\rangle}{\langle I\rangle},
$$

Finally, we have

$$
R(\Delta \mathbf{r})=\langle I\rangle^{2}\left(1+\|\rho(\Delta \mathbf{r})\|^{2}\right)
$$

or

$$
\Gamma(\Delta \mathbf{r}) \equiv\|\rho(\Delta \mathbf{r})\|^{2}=\frac{R(\Delta \mathbf{r})}{\langle I\rangle^{2}}-1
$$

where $\langle I\rangle$ is the average echo signal intensity over the region of interest. Here $\|\rho(\Delta \mathbf{r})\|^{2}$ is called the normalized correlation of the echo signal intensities and is denoted by $\Gamma(\Delta \mathbf{r})$, which has the properties of $\Gamma(0)=1.0$ and $\Gamma(\infty)=0$.

Equations (3) and (3') show that there is a simple relationship between the correlation function of the complex echo signals and the correlation function of the signal intensities, which is directly related to the speckle in B-mode images.

B. Application to Clinical Scanners. The instruments for 3D ultrasonic imaging currently are linear curved, linear, or other array scanners; here, a 1D linear array will be considered. To further simplify our analysis, we confine our measurements to the focal zone or Fraunhofer region of the array transducer. Applying the Huygens-Fresnel principle, the radiating surface of the transducer is considered as a collection of point sources

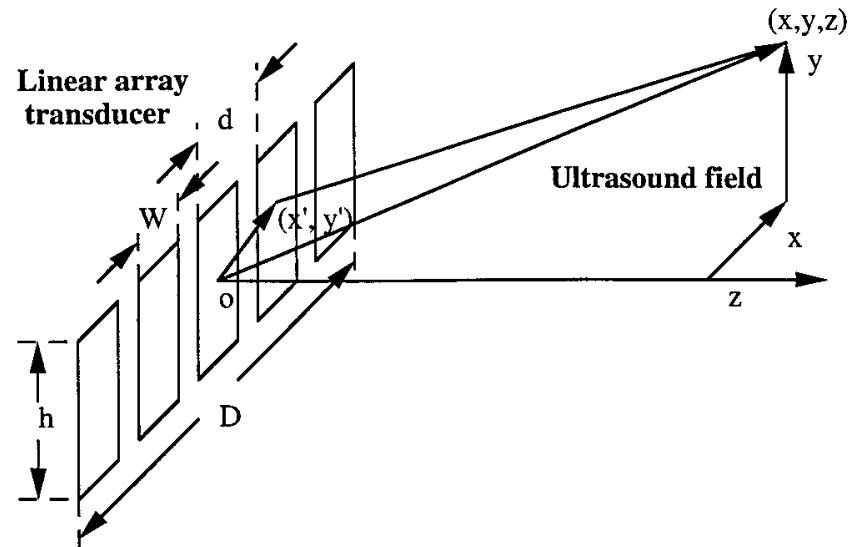

Figure 1. A transducer geometry for describing ultrasound beam patterns for a 1D linear array.

that oscillate sinusoidally. If the sources lie entirely within a simple plane $\left(x^{\prime}, y^{\prime}\right)$ as shown in Figure 1, the radiating surface is defined by an aperture distribution function [9]

$$
\begin{array}{r}
a\left(x^{\prime}, y^{\prime}\right)=\frac{1}{D w h / d}\left\{\left[\operatorname{rect}\left(\frac{x^{\prime}}{D}\right) \frac{1}{d} \operatorname{comb}\left(\frac{x^{\prime}}{d}\right)\right]\right. \\
\left.\otimes\left[\operatorname{rect}\left(\frac{\mathrm{x}^{\prime}}{\mathrm{w}}\right)\right]\right\} \operatorname{rect}\left(\frac{\mathrm{y}^{\prime}}{\mathrm{h}}\right)
\end{array}
$$

where $\left(x^{\prime}, y^{\prime}\right)$ is a point on the transducer surface, $w$ is the width of array elements, $d$ is the center-to-center distance between array elements, $D$ is the lateral dimension of the radiating aperture, $h$ is the height of array elements in the elevation direction, and functions

$$
\operatorname{comb}(x)=\sum_{i=-\infty}^{+\infty} \delta(x-i)
$$

and

$$
\operatorname{rect}(x)= \begin{cases}0 & |x|>\frac{1}{2} \\ 1 & |x| \leq \frac{1}{2}\end{cases}
$$

When the scan aperture is used to insonify and receive echoes from a medium containing randomly distributed fine particles, the complex echo signal is given by [9]

$$
U(\mathbf{r}) \sim C \frac{e^{2 i k z}}{z^{2}} h_{1}^{2}(x, y)
$$

where $k=2 \pi / \lambda_{o}$ is the ultrasound wave number, $\lambda_{o}$ is ultrasound wavelength at its central frequency, $z$ is a distance along the beam axis from the surface of the transducer to the field point, and $h_{1}(x, y)$ is called the directivity function. $h_{1}(x, y)$ is specified entirely in the $(x, y)$ plane (within the region of the interest) 


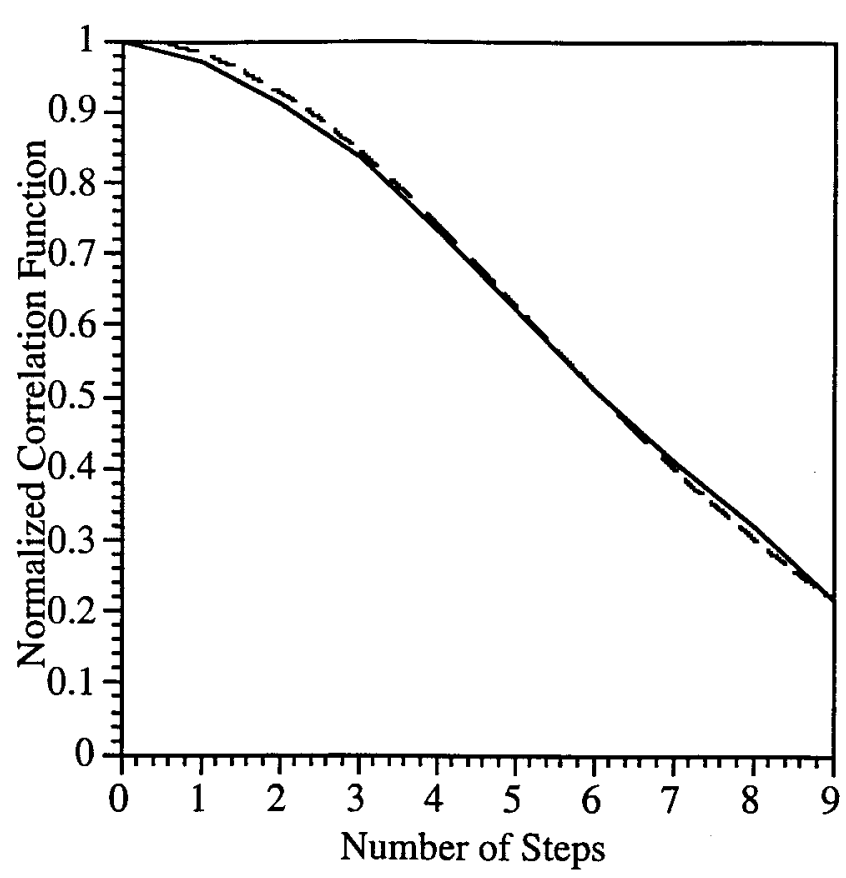

Figure 3. A typical experimental result of the normalized correlation function for the echo signal intensities (shown in a solid line) and its Gaussian fitting curve (shown in a dash line) when a transducer is slowly moved in the elevational direction. The step size is $0.2 \mathrm{~mm}$.

ROIs was then analyzed to compute the correlation between successive ROIs, i.e., ROI $1(i=1)$ correlation to ROI $2(i=2)$, ROI 2 to ROI 3, etc. for a series of one-step correlation function values. Then the process was repeated for all possible two, three, $\cdots n$ step correlation function values obtainable from the $i$ images. A correlation curve was created for any step sequence by plotting the zero-step through nine-step correlations. For this test, ROIs from groups of 10 images can be used to determine the slice separation for the center two slices of the 10, e.g., to position image 6 with respect to 5 use information from images $0-9$; for image 7 after 6 use image $1-10$, etc. This assumes a piecewise smooth motion for the scan head over an approximately $2 \mathrm{~mm}$ separation distance but this is a reasonable, first approach to the position estimate. In each case, the correlation function curve was fit to a Gaussian function using a least squares approach as shown in Equation ( $\left.7^{\prime}\right)$. The coefficients of these Gaussian functions are then used to position the images on an arbitrary grid space and correctly positioned images can then be displayed.

For a mechanical scanning with a uniform known 0.2-mm image slice separation (step size), a typical measurement result of the correlation function of the echo signal intensities as well as its Gaussian fitting curve are shown in Figure 3. Based on the coefficient in this Gaussian fitting curve and the point spread function in the elevational direction, the value of the step size can be estimated at that specific depth. Similarly, a group of the correlation functions are given in Figure 4 with the first curve calculated from the 10 slices starting at slice 1 , second curve starting with slice 2 , and so on. The step sizes were estimated based on the coefficients of the Gaussian fitting curves; the results are shown in Figure 5. The estimated step size is $(0.20 \pm 0.01)$

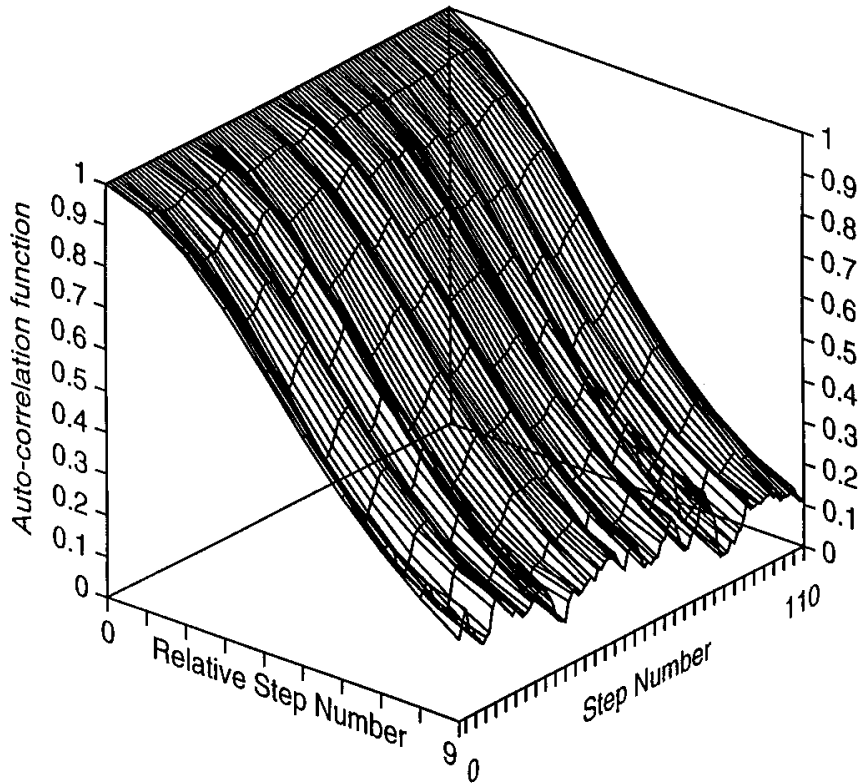

Figure 4. When the scan plane is automatically scanned through a tissue mimicking phantom in the elevational direction with uniform step sizes, the normalized correlation function of echo signal intensities as a function of different number of $0.2-\mathrm{mm}$ steps over a travel distance of $2.0 \mathrm{~cm}$.

$\mathrm{mm}$ over more than $20 \mathrm{~mm}$ travel distance of the scan head. The measurement error is approximately 5\%. This error could be caused by the statistical uncertainty of the correlation function estimates, the accuracy of the stepper motor (which is approximately $\pm 5 \mu \mathrm{m}$, or $\pm 2.5 \%$ based on a 200 - $\mu \mathrm{m}$ step size) and friction in the contact between the surface of the test phantom

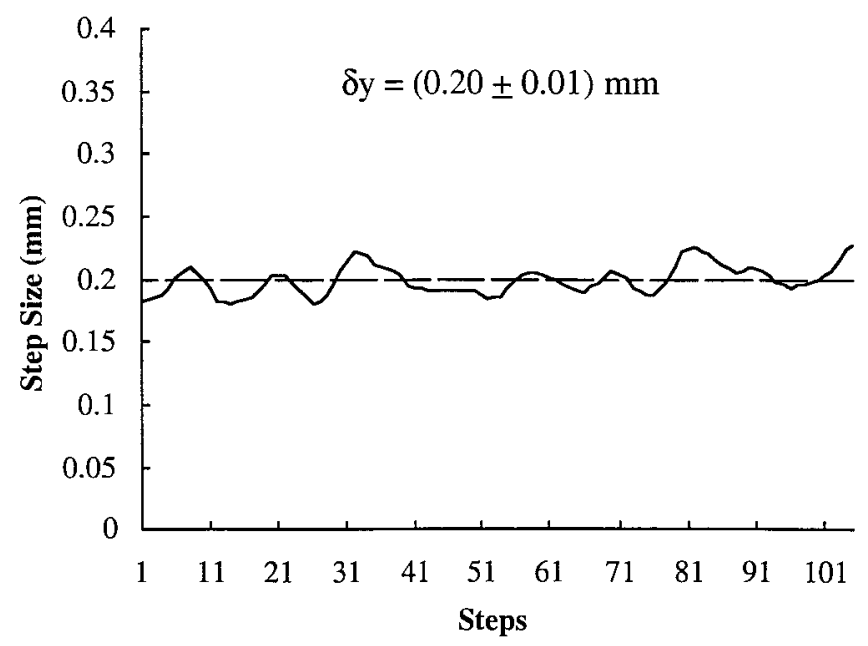

Figure 5. For a mechanical scanning with the uniform known 0.2$\mathrm{mm}$ step sizes (image slice separations), the step sizes estimated by using a series of Gaussian fitting curves to the measured correlation functions as a function of the step number is given by the solid line. The measurement result of the step size is $\delta=(0.20 \pm 0.01) \mathrm{mm}$. 
and the transducer, which might cause nonuniform scan-plane motion.

\section{DISCUSSION AND CONCLUSIONS}

As shown in this article, the results of measuring the decorrelation rate between a series of successive images can indicate the distance traveled in the simplest cases of pure elevational motion of the scan plane through an ideal medium. However, this case has not been addressed previously in medical imaging. There are numerous ways in which these concepts could be expanded to use correlation and decorrelation information to monitor scanplane motion. Many of these can be thought of simply by reversing the concepts used to measure tissue motion, although in current medical imaging cases, fluid and tissue tracking is done within the image plane where each tissue volume remains in the image plane for an extended number of frames. The following are a few example extensions and implementations for monitoring scanner motion using this decorrelation technique alone or in combination with other methods of motion detection.

\section{A. Scan-Head Motion Estimation in Less-than-Ideal Tis-} sues and Other Media. The current theory for the decorrelation of speckle addresses only the case where tissue can be modeled as a medium with acoustically uniform properties and containing many randomly distributed, pointlike scatterers. It may be possible to process the ultrasound data to minimize effects of specular and other scatterers violating the assumptions and to extrapolate detected motion over tissue segments where the decorrelation is anomalous. The best applications will be those in which random cumulative errors in scan position over long distances are not critical or can be corrected. In addition, one may not be restricted to motion estimate based on Gaussian statistics. Other statistical models for tissue may be used and even "training" sets of data for a particular application may prove more accurate for motion estimates.

\section{B. Monitoring Tilt and Rotation of the Transducer. Mea-} suring different motions at different locations within images should indicate transducer motion which is not uniform in the elevational direction. For example, tilting the transducer slightly can be detected by examining the decorrelation rate at different depths in the image using the depth-dependent point spread function. The difference in rate as a function of depth would indicate tilting of the scan head. In addition, transducer rotation might be tracked. For example, several different ROIs on either side of the central axis of the image could be monitored such that differences among these regions provide relative motion information. An important consideration here will be the required size of the ROI selected for the motion. A given ROI must be sufficiently large for a statistical measure of the motion, while the ROI must be small enough that the motion within the ROI can be considered as a single value. This is an area presently being examined.

C. On-the-Fly Processing of Position Information. By restricting the number of scan line locations transmitted and monitored for motion, it should be possible to measure motion rapidly enough to allow real-time scanning. The needed high-speed pulsing might come from power-mode color windows with multiple beamformers. It also should be possible to set motion criteria which will indicate when full images should be taken to provide an automated way of reducing the number of frames acquired in a 3D data set while assuring adequate sampling.

\section{Correction for Tissue Motion Such as Respiratory Mo-}

tion. This speckle decorrelation technique could also allow for the correction of respiratory motion when $3 \mathrm{D}$ data set are reconstructed. Elevational as well as in-plane corrections could be applied to keep an ROI stationary in the image. For nonuniform motion and deformation of the tissues, the size of the ROIs might be adaptive to maximize the precision of the correlation estimates, while providing adequate resolution numerous smaller ROIs might be used in conjunction with some spatial smoothing or warping software [11].

E. Use of Technique with Available Coarse Positioning. The currently described technique could also be used for fine positioning in conjunction with other positioning systems. The latter could monitor the long-range motion of the scan head, but might lack the fine spatial resolution required for the best 3D reconstructions. Such encoding devices include optical, mechanical, magnetic, and electric spark systems, all of which have been used in the past for 3D ultrasound imaging.

\section{F. Combination of 2D Speckle Tracking and Decorrela-} tion. One possible implementation would incorporate 2D speckle tracking [1] to monitor motion in the scan plane between adjacent slices and then measure the decorrelation result. The idea is that the 2D speckle tracking would identify the correct vertical and horizontal translation required for placing the adjacent slice with respect to the first. The decorrelation which then remained between the slices would be the result of the translation in the elevational direction and would be an improved estimate over assuming no vertical or horizontal motion. Therefore, one would create a $3 \mathrm{D}$ directional vector for the motion at a particular location in the image.

\section{G. Speed or Flexibility of Decorrelation Rate Determina-} tion. Use a threshold, rather than a Gaussian fitting function, to increase speed of decorrelation measurement. Alternatively, to maintain noise smoothing with more flexibility, use an adaptive fitting function, rather than a fixed Gaussian. With an adaptive fitting, it may be possible, for example, to correct for periodic motion, such as cardiovascular tissue motion.

In summary, a method is described for determining the scanplane relative positions in the elevational direction using the decorrelation of speckle signal intensities in B-mode images, which are not due to motion within the scan plane. The method was tested using finely spaced scans in a tissue-mimicking phantom containing randomly distributed, fine, solid particles. The experimental results are in rather good agreement with the known step size, i.e. only $5 \%$ rms deviation. Useful implementation will depend on development and testing for more general and challenging conditions, including considerations of various artifacts in speckle tracking such as cumulative error and misinterpreted motion [12]. Experiments are currently being conducted in actual tissue to determine the efficacy of the technique and potential sources of error. 


\section{APPENDIX}

We consider a complex random process, e.g., the complex echo signal, including both amplitude and phase information. We are interested in the correlation function of the complex echo signals between two subsequent scan plane positions. The complex echo signals are given by [8]

$$
U(\mathbf{r})=U_{r}(\mathbf{r})+i U_{i}(\mathbf{r})
$$

and

$$
U(\mathbf{r}+\Delta \mathbf{r})=U_{r}(\mathbf{r}+\Delta \mathbf{r})+i U_{i}(\mathbf{r}+\Delta \mathbf{r})
$$

where $\mathbf{r}$ and $\mathbf{r}+\Delta \mathbf{r}$ are the positions within an ROI related to the transducer scan head. The completely random signals are taken to have zero mean and to have the same variances of the real and imaginary parts. Then the autocorrelation function of the resulting signal is given by

$$
\rho(\Delta \mathbf{r})=\left\langle U(\mathbf{r}) U^{*}(\mathbf{r}+\Delta \mathbf{r})\right\rangle
$$

where * refers to the complex conjugation, and displacement between points in the two scan planes is given by $\Delta \mathbf{r}$. If this field is simply read out or scanned by an imaging system with a point spread function (psf) $h(\mathbf{r})$, the resulting complex signal $U(\mathbf{r})$ is related to the original field $\alpha(\mathbf{r})$ by

$$
U(\mathbf{r})=h(\mathbf{r}) \otimes \alpha(\mathbf{r})
$$

where $\otimes$ is a convolution operation and $\alpha(\mathbf{r})$ depends on the properties of the medium. The correlation function of the resulting processing, $\rho(\Delta \mathbf{r})$ is then directly given by [7]

$$
\rho(\Delta \mathbf{r})=h(-\Delta \mathbf{r}) \otimes \rho_{\alpha}(\Delta \mathbf{r}) \otimes h^{*}(\Delta \mathbf{r})
$$

where $\rho_{\alpha}(\Delta \mathbf{r}) \equiv\left\langle\alpha(\mathbf{r}) \alpha^{*}(\mathbf{r}+\Delta \mathbf{r})\right\rangle$. Equation (A3) shows that the correlation function depends on the displacement of the beam related to scatterers in the ROI.

A typical example is the acoustic scattering from a random collection of $N$ scatterers in and extending well beyond a resolution cell or volume. These $N$ scatterers can be divided into $L$ sets, each set containing $N_{l}$ scatterers $\left(\sum_{l=1}^{L} N_{l}=N\right)$, and all having the same displacement, $\Delta \mathbf{r}_{l}$ from the previous set. In that case, we have

$$
\alpha(\mathbf{r})=\sum_{i=1}^{N}\left|a_{i}\right| e^{i \phi_{i}}
$$

and

$$
\alpha(\mathbf{r}+\Delta \mathbf{r})=\sum_{j=1}^{N}\left|a_{j}\right| e^{i \phi_{j}}
$$

where $a_{i}$ is the magnitude of the scattering amplitude of the $i$ th scatterer and there is correlation neither among the scatter phase $\phi_{i}$ in the first scattering volume nor among those in the second scattering volume nor between the scattering volumes. Then the correlation function of $\alpha(\mathbf{r})$ is

$$
\begin{aligned}
\left\langle\alpha(\mathbf{r}) \alpha^{*}(\mathbf{r}+\Delta \mathbf{r})\right\rangle & =\sum_{i=1}^{N} \sum_{j=1}^{N}\left\langle\left|a_{i}\right|\left|a_{j}\right| e^{i\left(\phi_{i}-\phi_{j}\right)}\right\rangle \\
& =\sum_{l=1}^{L}\left\{N_{l}\left|a_{l}\right|^{2} \delta\left(\Delta \mathbf{r}_{l}\right)\right\}
\end{aligned}
$$

since all phases average out unless they match up identically. Here, $\delta\left(\Delta \mathbf{r}_{l}\right)$ is the Dirac delta function.

Finally, the correlation function of the readout or scanned process is simplified to

$$
\rho(\Delta \mathbf{r})=\sum_{l=1}^{L} \rho_{l}\left(\Delta \mathbf{r}_{l}\right)
$$

where $\rho_{l}\left(\Delta \mathbf{r}_{l}\right)$ is given by

$$
\rho_{l}\left(\Delta \mathbf{r}_{l}\right)=N_{l}\left\|a_{l}\right\|^{2}\left\{h\left(-\Delta \mathbf{r}_{l}\right) \otimes h^{*}\left(\Delta \mathbf{r}_{l}\right)\right\}
$$

In the far field or the focal zone of the transducer, the psf can be approximately separated into the transverse component due to the diffraction limited beam, and the axial component due to the ultrasonic pulse,

$$
h(\Delta \mathbf{r}) \approx h_{t}\left(r_{t}\right) h_{r}\left(r_{r}\right)
$$

where $h_{t}\left(r_{t}\right)$ and $r_{t}$ are the transverse components of the psf and the displacement of the scatterers, and $h_{r}\left(r_{r}\right)$ and $r_{r}$ are the axial components of the psf and the displacement of scatterers. As a simple example, when all scatterers are in motion (relative to the scan head) in the lateral direction, the correlation function of the complex echo signal will just depend on the transducer beam properties. When the scatterers are in motion in the axial direction, that function will depend on the ultrasonic pulses.

\section{ACKNOWLEDGMENTS}

The authors are grateful to Aaron Moskalik for constructing the mechanical scanning system and developing the algorithms for data acquisition and analysis. This work was supported by in part by USPHS Grant R01 CA55076, and by Grant DAMD17-94-54144 from the USAMRDC.

\section{REFERENCES}

1. E. J. Chen, W. K. Jenkins, and W. D. O'Brien, Jr. “The impact of various imaging parameters on ultrasonic displacement and velocity estimates," IEEE Trans. Ultrasonics Ferroelect. Freq. Control UFFC-41, 293-301 (1994).

2. R. F. Wagner, S. W. Smith, J. M. Sandrik, and H. Lopez. "'Statistics of speckle in ultrasound B-scans,' IEEE Trans. Sonics Ultrasonics SU-30, 156-163 (1983).

3. S. W. Smith, R. F. Wagner, J. M. Sandrik, and H. Lopez. "Low contrast detectability and contrast/detail analysis in medical ultrasound," IEEE Trans. Sonics Ultrasonics SU-30, 164-173 (1983).

4. G. E. Trahey, S. W. Smith, and O. T. Von Ramm. "'Speckle pattern correlation with lateral aperture translation: experimental results and implications for spatial compounding,' IEEE Trans. Ultrasonics Ferroelect. Freq. Control UFFC-33, 257-264 (1986).

5. M. O'Donnell and S. D. Silverstein. "Optimum displacement for compound image generation in medical ultrasound,' IEEE Trans. Ultrasonic Ferroelect. Freq. Control UFFC-35, 470-476 (1988). 
6. R. S. Adler, J. M. Rubin, J. B. Fowlkes, P. L. Carson, and J. E. Pallister. "Ultrasonic estimation of tissue perfusion: a stochastic approach,"' Ultrasound Med. Biol. 21, 493-500 (1995).

7. L. Nock and G. E. Trahey. "Phase aberration correction in medical ultrasound using speckle brightness as a quality factor," J. Acoust. Soc. Am. 85, 1819-1833 (1989).

8. R. F. Wagner, M. F. Insana, and D. G. Brown. "Statistical properties of radio-frequency and envelope-detected signals with applications to medical ultrasound,"' J. Opt. Soc. Am. 4, 910-922 (1987).

9. M. F. Insana, T. J. Hall, and L. T. Cook. "Backscatter coefficient estimation using array transducers," IEEE Trans. Ultrasound Ferroelect. Freq. Control UFFC-41, 714-723 (1994).
10. A. Moskalik, P. L. Carson, C. R. Meyer, J. B. Fowlkes, J. M. Rubin, and M. A. Roubidoux. "Registration of 3D compound ultrasound scans of the breast for refraction and motion correction," Ultrasound Med. Biol. 21, 769-778 (1995).

11. B. Kim, J. L. Boes, K. A. Frey, and C. R. Meyer, "Mutual information for automated multimodal image warping," in Proceedings of Visualization and Biomedical Computing: Lecture Notes in Computer Science, Vol. 1131. Springer-Verlag, Hamburg, Germany, 1996, pp. 349-354.

12. F. Kallel, M. Bertrand, and J. Meunier. "Speckle motion artifact under tissue rotation," IEEE Trans. Ultrasound Ferroelect. Freq. Control UFFC-41, 205-258 (1994). 